\title{
RIESZ BASES AND POSITIVE OPERATORS ON HILBERT SPACE
}

\author{
JAMES R. HOLUB
}

Received 2 February 2002

\begin{abstract}
It is shown that a normalized Riesz basis for a Hilbert space $H$ (i.e., the isomorphic image of an orthonormal basis in $H$ ) induces in a natural way a new, but equivalent, inner product on $H$ in which it is an orthonormal basis, thereby extending the sense in which Riesz bases and orthonormal bases are thought of as being the same. A consequence of the method of proof of this result yields a series representation for all positive isomorphisms on a Hilbert space.
\end{abstract}

2000 Mathematics Subject Classification: 46B15, 46C05, 47B65.

1. Introduction. Let $H$ denote a Hilbert space (assumed real, for notational convenience) with inner product $(\cdot, \cdot)$ and let $\left\{x_{i}\right\}$ be a basis for $H$ having coefficient functionals $\left\{f_{i}\right\}$ denoted by $\left\{x_{i}, f_{i}\right\}$. We say that $\left\{x_{i}, f_{i}\right\}$ is a Riesz basis for $H$ if it has the property that $\sum a_{i} x_{i}$ converges in $H$ if and only if $\left\{a_{i}\right\}$ is in the sequence space $l^{2}$. Equivalently, $\left\{x_{i}, f_{i}\right\}$ is a Riesz basis for $H$ if and only if there is an isomorphism $U$ on $H$ and some orthonormal basis $\left\{\phi_{i}\right\}$ for $H$ so that $U \phi_{i}=x_{i}$ for all $i$, implying that Riesz bases and orthonormal bases are the "same" in linear-topological terms, but differ in geometrical ones due to the additional orthogonality relations between basis vectors in an orthonormal basis that is lacking in a Riesz basis. The result below (Theorem 2.1) shows that this is, in a sense, an artificial distinction by showing that every Riesz basis, in fact, is an orthonormal basis for $H$ under a different, but equivalent, inner product.

\section{Main results}

THEOREM 2.1. Let $\left\{x_{i}, f_{i}\right\}$ be a normalized Riesz basis for a Hilbert space $H$. Then there is an equivalent inner product on $H$ in which $\left\{x_{i}\right\}$ is an orthonormal basis for $H$ under the norm induced by this inner product.

Proof. If $x$ and $y$ are any two vectors in $H$, then the sequences $\left\{\left(f_{i}, x\right)\right\}$ and $\left\{\left(f_{i}, y\right)\right\}$ are in $l^{2}$, implying that $\sum\left(f_{i}, x\right)\left(f_{i}, y\right)$ converges. Clearly, the bilinear form on $H \times H$, defined by $\langle x, y\rangle=\sum\left(f_{i}, x\right)\left(f_{i}, y\right)$, is then an inner product on $H$ for which $\left\langle x_{i}, x_{j}\right\rangle=d_{i j}$ for all $i$ and $j$, in which $\left\{x_{i}\right\}$ is an orthonormal set that is also complete, since if $\left\langle x_{n}, x\right\rangle=0$ for all $n$, then $0=\sum\left(f_{i}, x_{n}\right)\left(f_{i}, x\right)=$ $\left(f_{n}, x\right)$ for all $n$; that is, $0=\sum\left(f_{i}, x_{n}\right)\left(f_{i}, x\right)$ by definition of the new inner product for all $n$, implying that $\left(f_{n}, x\right)=0$ for all $n$, and hence that $x=0$. 
As usual, the inner product $\langle\cdot, \cdot\rangle$ defines a norm $\|\cdot\|_{1}$ on $H$ by $\|x\|_{1}^{2}=$ $\langle x, x\rangle=\sum\left|\left(f_{i}, x\right)\right|^{2}$. Since $\left\{x_{i}\right\}$ is a Riesz basis, there is an isomorphism $U$ on $H$ that maps each vector $\phi_{i}$ in an orthonormal basis $\left\{\phi_{i}\right\}$ for $H$ to the vector $x_{i}$, implying that the isomorphism $V=\left(U^{*}\right)^{-1} U^{-1}$ on $H$ maps $x_{i}$ to $f_{i}$ for all $i$. Since, for any $x$ in $H,\langle x, x\rangle=\sum\left(f_{i}, x\right)\left(f_{i}, x\right)=\left(\sum\left(f_{i}, x\right)\left(V x_{i}, x\right)\right)=$ $\sum\left(f_{i}, x\right)\left(V x_{i}, x\right)=\left(V\left[\sum\left(f_{i}, x\right) x_{i}\right], x\right)=(V x, x)$, we see that $(V x, x)=\sum \mid\left(f_{i}\right.$, $x)\left.\right|^{2}=\|x\|_{1}^{2}$ for all $X$ in $H$, so $V$ is a positive operator. If we let $W$ denote the positive square root of $V$, then $W$ is also an isomorphism on $H$ so that, for any $x$ in $H$, we have $\|x\|_{1}^{2}=(V x, x)=(W x, W x)=\|W x\|^{2} \leq\|w\|^{2}\|x\|^{2}$. In the same way, we see that $\|x\|_{1}^{2} \leq\left\|W^{-1}\right\|^{2}\|x\|^{2}$, and it follows that the new norm $\|\cdot\|_{1}$ is equivalent to the original norm on $H$. In particular, $H$ is then complete under the new norm, hence a Hilbert space, in which $\left\{x_{i}\right\}$ is then an orthonormal basis, being an orthonormal set, that is complete in the new inner product.

3. Positive operators. In the proof above we used the fact that if $\left\{x_{i}, f_{i}\right\}$ is a Riesz basis for a Hilbert space $H$, then the operator $U$ on $H$, mapping $x_{i}$ to $f_{i}$, is a positive isomorphism on $H$. It is interesting to note that, in fact, every positive isomorphism on $H$ is such an operator for some Riesz basis in $H$, thereby providing a representation for all positive isomorphisms $U$ on a Hilbert space.

THEOREM 3.1. An operator $U$ on a Hilbert space on $H$ is a positive isomorphism if and only if $U$ is of the form $U=\sum f_{i} \otimes f_{i}$ for some Riesz basis $\left\{x_{i}, f_{i}\right\}$ for $H$ (i.e., $U x_{i}=f_{i}$ for all $i$ ).

Proof. If $U=\sum f_{i} \otimes f_{i}$ for some Riesz basis $\left\{x_{i}, f_{i}\right\}$ for $H,\left\{\phi_{i}\right\}$ is an orthonormal basis for $H$, and $T$ is the isomorphism on $H$ mapping $\phi_{i}$ to $f_{i}$ for all $i$, then $U=\sum T \phi_{i} \otimes T \phi_{i}=T T^{*}$, a positive isomorphism on $H$.

Conversely, if $U$ is any positive isomorphism on $H$, then $W$, the positive square root of $U$, is also an isomorphism on $H$. If we set $f_{i}=W \phi_{i}$ for some orthonormal basis $\left\{\phi_{i}\right\}$, then $\left\{f_{i}\right\}$ is a Riesz basis for $H$ so that, for any $x$ in $H$, we have $U x=W^{2} x=W\left[\sum\left(\phi_{i}, W x\right) \phi_{i}\right]=W\left[\sum\left(W \phi_{i}, x\right) \phi_{i}\right]=\sum\left(f_{i}, x\right) W \phi_{i}=$ $\sum\left(f_{i}, x\right) f_{i}$. That is, $U=\sum_{i} f_{i} \otimes f_{i}$ and the proof is complete.

James R. Holub: Department of Mathematics, Virginia Polytechnic Institute and State University, Blacksburg, VA 24061-0123, USA

E-mail address: holubj@math.vt.edu 


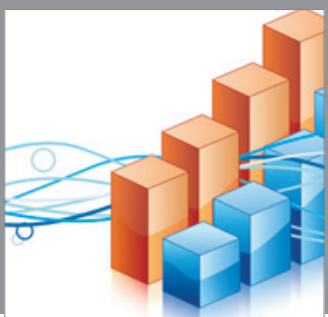

Advances in

Operations Research

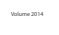

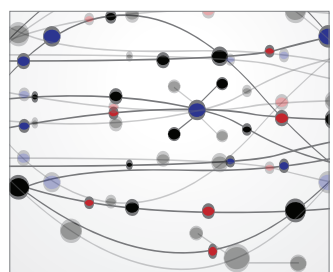

\section{The Scientific} World Journal
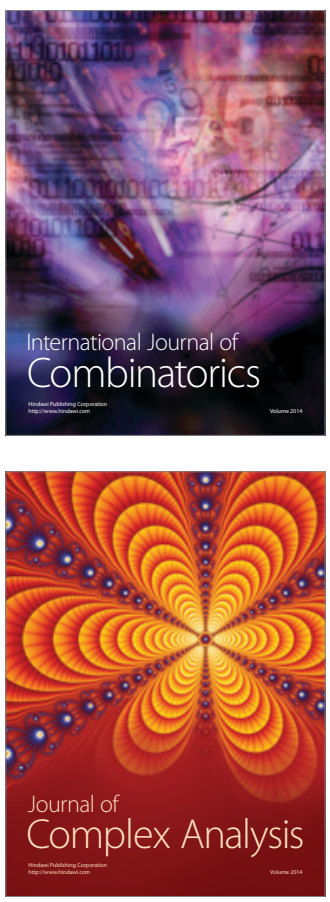

International Journal of

Mathematics and

Mathematical

Sciences
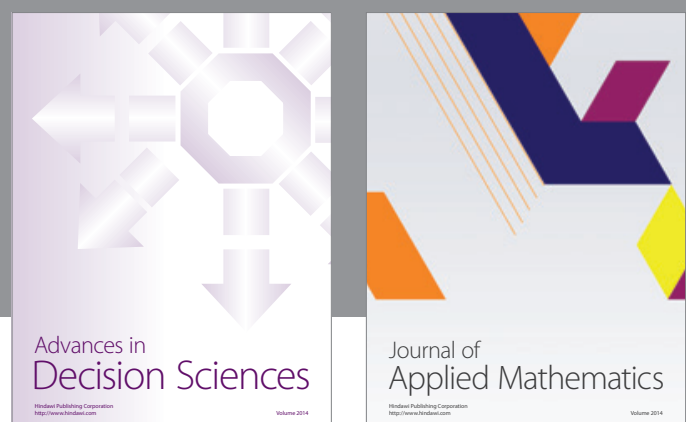

Journal of

Applied Mathematics
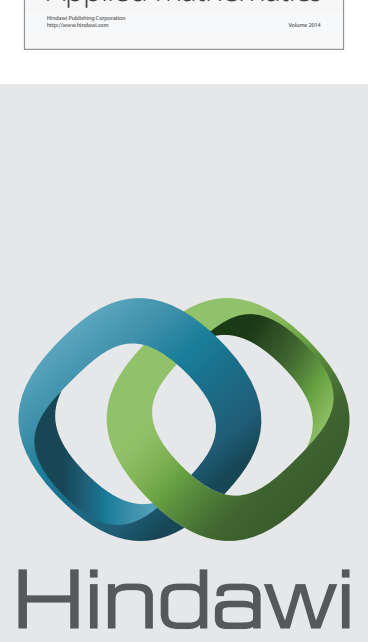

Submit your manuscripts at http://www.hindawi.com
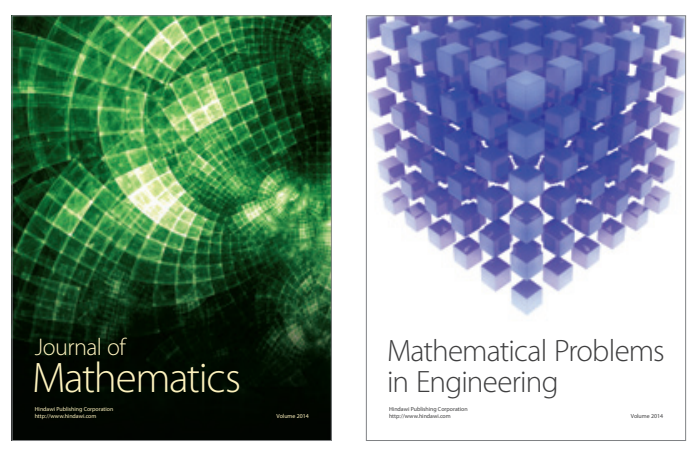

Mathematical Problems in Engineering
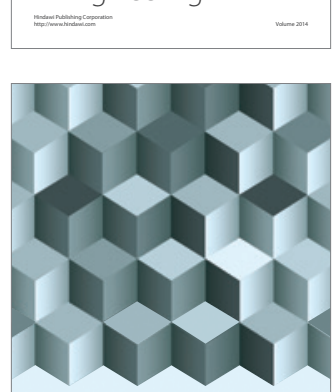

Journal of

Function Spaces
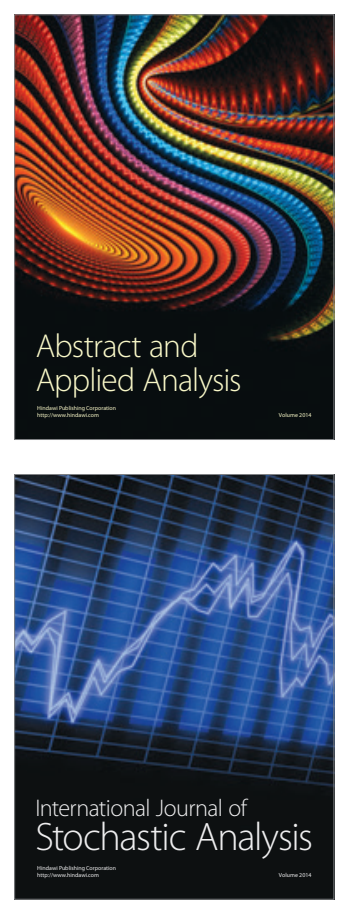

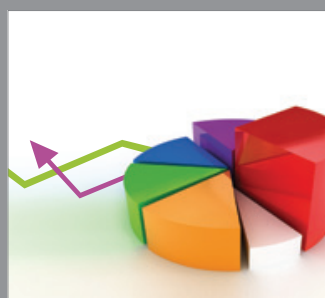

ournal of

Probability and Statistics

Promensencen
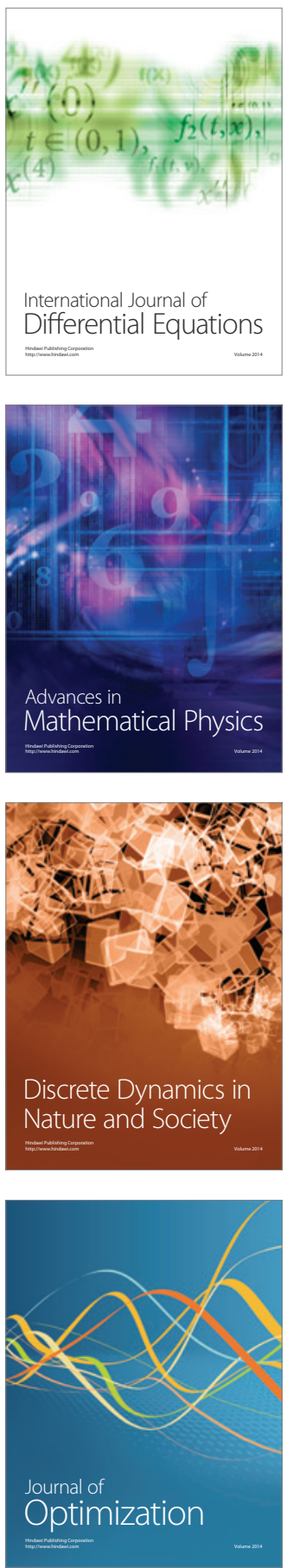